\title{
Genetic diversity of HLA-DRB1 alleles in the Tujia population of Wufeng, Hubei Province, China
}

\author{
X.P. Qiu ${ }^{1}$, L. Zhang ${ }^{1 *}$, F.Y. Zeng ${ }^{5 *}$, Y. Wen ${ }^{2}$, C. Li ${ }^{3}$, L.X. Qiu ${ }^{1}$, D.X. Cheng ${ }^{4}$ \\ and $\mathrm{X} . \mathrm{X} . \mathrm{Wu}^{1}$ \\ ${ }^{1}$ Institute of Virology, Medical School of Wuhan University, Wuhan, \\ Hubei, China \\ ${ }^{2}$ Medical School of Hubei University of Science and Technology, \\ Hubei, China \\ ${ }^{3}$ Wuhan Nursing Home Hot Spring Rehabilitation Sanitarium Area, \\ Xianning, Hubei, China \\ ${ }^{4}$ Department of Medicine and Medical Biophysics, University of Toronto, \\ Toronto, Ontario, Canada \\ ${ }^{5}$ The Clinical Laboratory, Wuhan Hospital, Wuhan, China \\ *These authors contributed equally to this study. \\ Corresponding author: X.X. Wu \\ E-mail: qiuxp8488@sohu.com \\ Genet. Mol. Res. 13 (3): 4756-4765 (2014) \\ Received August 28, 2013 \\ Accepted January 10, 2014 \\ Published July 2, 2014 \\ DOI http://dx.doi.org/10.4238/2014.July.2.5
}

\begin{abstract}
We established a genetic database by investigating human leukocyte antigen (HLA)-DRB1 allelic frequencies in a disease-association study in the Tujia population in Wufang, Hubei, China. The allele frequencies of the HLA-DRB1 locus in 262 healthy, unrelated Tujia individuals living in the Wufeng region of the Hubei Province were analyzed using the Luminex HLA sequence-specific oligonucleotide method with a WAKFlow HLA typing kit. A total of 13 alleles were detected at the HLA-DRB1 locus. HLA-DRB1*09 was the most common allele $(22.52 \%)$, followed by DRB $1 * 08$ and
\end{abstract}


DRB1*15 (11.07\%), and DRB1*12 and DRB1*04 (10.69\%). These data were compared with the results obtained for 10 other ethnic groups living in other regions as well as to Han groups using neighbor-joining dendrograms and principal component analysis. The results showed that the Tujia population has a close genetic relationship with the Middle Han population at the HLA-DRB1 locus. This information will be useful for HLA-DRB1-linked disease-association studies.

Key words: Alleles; HLA-DRB1; Principal component analysis; Neighbor-joining dendrograms

\section{INTRODUCTION}

The Tujia ethnic group, a minority group with a long history, mainly inhabits the Wuling mountain area on the borders between the Hunan, Hubei, Sichuan, and Guizhou provinces of China. Wufeng County, in which most of the Tujia population of 207,700 resides, is located in the southwestern region of the Hubei Province neighboring the Hunan Province. This area covers $2072 \mathrm{~km}^{2}$ of mountain area and extends into part of the Wuling mountain range. This mountain area comprises the easternmost Yunnan and Guizhou high plateau with typical karst topography. There are many karst caves; subterranean drainage occurs, and the land falls from west to east, with $86.3 \%$ of the land sitting higher than $500 \mathrm{~m}$ above sea level and $44.8 \%$ of the mountain areas sitting at more than $1200 \mathrm{~m}$ above sea level. The highest mountain in Wufeng is Baiyizhai Mountain, with an altitude of $2320.3 \mathrm{~m}$ above sea level, while the lowest point, at only $150 \mathrm{~m}$ above sea level, is located at Qiaohe Gorge east of the Yuyang River. Wufeng contains many gullies, ravines, and mountain chains. Small areas of flatland are scattered among the mountainside, while larger areas of plains with nearly 667 hectare located in the east at Yuyang Guan (lower mountain valley), in the center of Changleping (middle area), and west of WanTan (high mountain area). Wufeng natural topography provides a natural barrier to population movement, making this mountainous area an ideal region for human genetic research.

The group of genes encoding human leukocyte antigen (HLA) is found on chromosome 6 (6p12.3). Several previous studies have shown that the HLA system, the most complicated immune genetic system, has the highest rate of genetic polymorphisms among the entire human genome (Bodmer, 1987). The major histocompatibility complex (MHC) locus is increasingly used as a genetic marker to investigate the genetic structure of humans and to determine the relationship between closely related species and their evolving history. During natural selection, $\mathrm{MHC}$ genes are typically retained in the process of species development; thus, MHC classification is also widely used in matching organs for transplantation and in disease-related field research. The high genetic polymorphism rates of HLA genes results in significant differences in allele frequency between populations from different species, nationalities, and population areas. These polymorphisms also play an important role in individual recognition in the fields of forensics and in human genetic research, such as in determining an ethnic group origin, evolution, migration, and merging with other ethnic groups (Ivanova et al., 2002; Shankarkumar et al., 2003).

A total of 262 non-related Tujias from the Hubei Wufeng area were involved in this study; their HLA-DRB1 locus was genotyped and their allele frequency at the HLA-DRB1 lo- 
cus was compared with that of 10 other nationalities. The Tujias evolutionary position among that of other ethnic groups was established. These results will help future investigations on the genetic background of HLA-DRB1 alleles and their related diseases.

\section{MATERIAL AND METHODS}

\section{Sample collection}

This project was approved by the Medical Ethics Committee of Wuhan University, China. The study population included 262 healthy, unrelated donors from the 51 towns of the Wufeng region in China. Some of the towns are shown in Figure 1. All of these individuals' ancestors had been born and lived in the Wufeng region of Hubei Province for at least 3 generations. After obtaining informed consent from all participants, blood samples were collected.

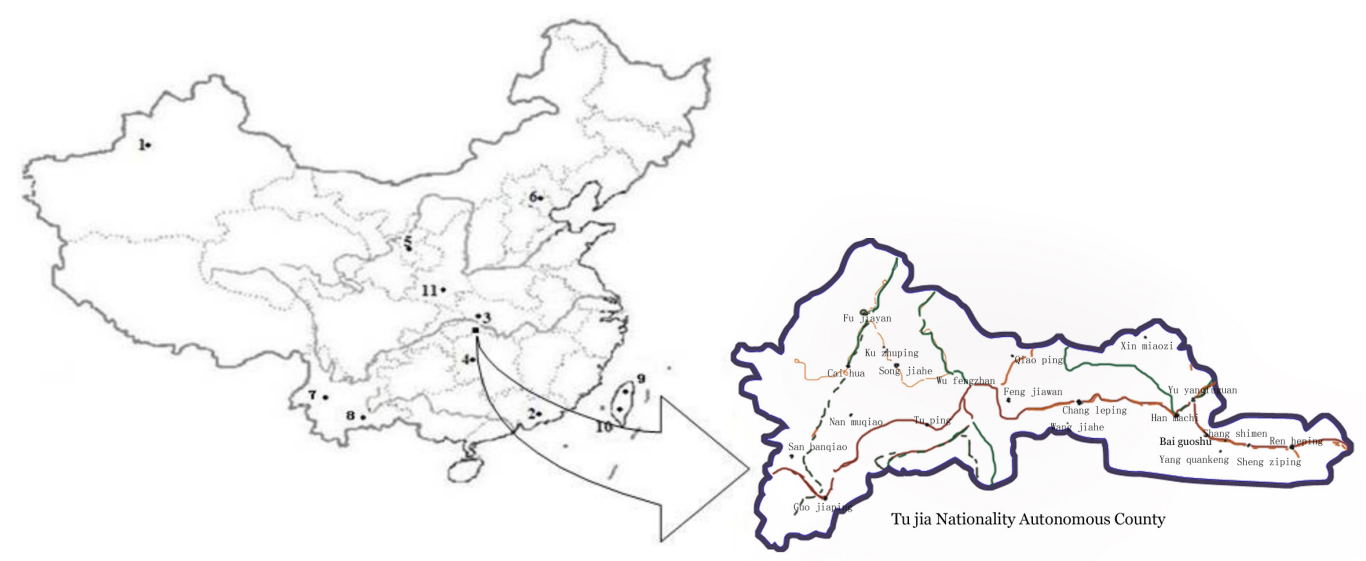

Figure 1. Tujia Nationality Autonomous County. Map showing the sites of the populations used in this study and 21 towns of Tujia Nationality Autonomous County of Wufeng. 1 = Uyghur, 2 = Southern-Han, 3 = Tujia, 4 = Miao, $5=$ Hui, $6=$ Northern-Han, $7=$ Bulang, $8=$ Hani, $9=$ Minnan, $10=$ Taiwan-Aborigines, $11=$ Middle-Han.

The main industry in Wufeng County is farming, although this area lacks significant natural resources. The per capita yearly income is 1400 Yuan; 57,496 people lived in poverty with an annual income of less than 625 Yuan from 1998 to 2002. Thus, Wufeng County is considered a poverty-stricken county in Hubei Province.

\section{DNA extraction}

Genomic DNA was isolated from whole blood using a Genomic DNA Isolation Kit 
(Aidlab Biotechnologies Co. Ltd.; Beijing, China), according to manufacturer instructions. Blood was collected into an ethylenediamine tetraacetic acid (EDTA)-containing tube and gently shaken until thoroughly mixed. Nine hundred microliters cell lysis solution were added to a sterile $1.5-\mathrm{mL}$ centrifuge tube, and then $300 \mu \mathrm{L}$ blood were added to the tube containing the cell lysis solution and the tube was inverted 5-6 times to mix. The mixture was incubated for $10 \mathrm{~min}$ at room temperature and then centrifuged at $12,000 \mathrm{~g}$ for $20 \mathrm{~s}$; as much supernatant as possible was removed and discarded. The tube was vortexed vigorously until the remaining pellet of white blood cells was completely resuspended and separated. An additional $300 \mu \mathrm{L}$ cell lysis solution was added to the resuspended cells and the tube was inverted completely and vortexed vigorously for $10 \mathrm{~s}$ until the clumps were disrupted. Next, $800 \mu \mathrm{L}$ protein precipitation solution was added and the mixture was vortexed vigorously for $25 \mathrm{~s}$. After centrifugation at 13,000 $\mathrm{g}$ for $5 \mathrm{~min}$, a dark brown protein pellet was visible. Approximately $300 \mu \mathrm{L}$ supernatant were carefully transferred to a new $1.5-\mathrm{mL}$ centrifuge tube and $300 \mu \mathrm{L}$ room temperature $70 \%$ ethanol were added. The tube was gently inverted 30 times or until the pellet was almost white, and then centrifuged at 12,000 $\mathrm{g}$ for $1 \mathrm{~min}$. The supernatant was carefully removed, leaving a white pellet of DNA. This pellet was washed by adding $1 \mathrm{~mL} \mathrm{70 \%} \mathrm{ethanol,} \mathrm{inverting} \mathrm{the} \mathrm{tube} \mathrm{several} \mathrm{times,} \mathrm{and} \mathrm{then} \mathrm{centrifuged} \mathrm{at} \mathrm{12,000}$ $g$ for $1 \mathrm{~min}$ and the ethanol was carefully aspirated. The pellet was air-dried for several minutes, and then $100 \mu \mathrm{L}$ DNA rehydration solution was added to the tube. The DNA was dissolved by gently tapping the tube and then incubating the solution at $65^{\circ} \mathrm{C}$ for $30-60 \mathrm{~min}$ at room temperature, or at $4^{\circ} \mathrm{C}$ overnight. The DNA was stored at $2-8^{\circ} \mathrm{C}$ or at $-20^{\circ} \mathrm{C}$ for longterm storage. The concentration of DNA was $40-100 \mathrm{ng} / \mathrm{L}$, with a purity of the extracted DNA showing an optical density of 1.6-1.85.

\section{DNA typing of the HLA locus}

HLA-DRB1 genotyping was performed on a Multi-Analyte Profiling system (xMAP) (Luminex HLA-SSO; Shanghai, China) using a WAKFlow HLA typing kit according to manufacturer instructions (Qiu et al., 2011).

\section{Statistical and phylogenetic analysis}

Allelic frequencies of the HLA-DRB1 locus were estimated using the direct counting method. Principal component analysis (PCA) was performed using the SPSS 13.0 software (SPSS Inc.; Chicago, IL, USA), and was performed in different populations using the allelic frequencies of the HLA-DRB1 locus as parameters. Phylogenetic trees (dendrograms) were constructed based on allelic frequencies using the neighbor-joining method with Nei genetic distances using the Phylip phylogeny program (http://evolution.gs.washington.edu/phylip. html) (Saitou and Nei, 1987).

\section{RESULTS}

\section{Hardy-Weinberg tests of the HLA-DRB1 locus}

The frequencies of HLA-DRB1 genes were tested for Hardy-Weinberg equilibrium 
using the Arlequin 3.11 software (http://anthro.unige.ch/software/arlequin/) (Excoffier and Slatkin, 1995). The HLA-DRB1 gene distributions of the Tujia nationality were found to be in Hardy-Weinberg equilibrium $(\mathrm{P}>0.05)$.

\section{Allele frequencies}

The frequencies of the HLA-DRB1 alleles are shown in Table 1. We identified a total of 13 alleles at the HLA-DRB1 locus in the Tujia population. Five alleles showed frequencies exceeding 10\% [HLA-DRB1*09 (22.52\%), DRB1*08 and DRB1*15 (11.07\%), DRB1*04 and DRB1*12 (10.69\%)], with a cumulative frequency of $66.04 \%$. No rare HLA-DRB1 alleles (allele frequencies $<1 \%$ ) were found in the Tujia population of the Wufeng region.

\begin{tabular}{|c|c|c|}
\hline HLA-DRB1 alleles & $\mathrm{N}(262 \times 2=524)$ & Allelic frequency $(\%)$ \\
\hline DRB $1 * 01$ & 15 & 2.86 \\
\hline DRB $1 * 04$ & 56 & 10.69 \\
\hline DRB $1 * 07$ & 20 & 3.82 \\
\hline DRB $1 * 08$ & 58 & 11.07 \\
\hline DRB $1 * 09$ & 118 & 22.52 \\
\hline DRB1*3 (17) & 20 & 3.82 \\
\hline $\mathrm{DRB} 1 * 10$ & 11 & 2.1 \\
\hline DRB $1 * 11$ & 37 & 7.06 \\
\hline DRB $1 * 12$ & 56 & 10.69 \\
\hline DRB $1 * 13$ & 27 & 5.15 \\
\hline DRB $1 * 14$ & 41 & 7.82 \\
\hline DRB $1 * 15$ & 58 & 11.07 \\
\hline DRB $1 * 16$ & 7 & 1.34 \\
\hline
\end{tabular}

\section{PCA and phylogenetic tree analysis}

Allele frequency data were compared with data from 10 other populations examined in previous studies (Shaw et al., 1999; Chen et al., 2007; Shen et al., 2010; Shi et al., 2010). The Uyghur, Southern Han, Northern Han, Middle-Han, Min-nan, Taiwan aborigines, Miao, Hui, Bulang, and Hani were blood donors. Nei genetic distances were calculated based on the allele frequencies of different populations. The resulting phylogenetic tree is shown in Figure 2. The genetic structure of the Tujia population was found to be closest to that of the MiddleHan population. PCA of the 11 ethnic groups was performed based on the allelic frequencies of the HLA-DRB1 locus, as shown in Figure 3. Two major clusters were identified in the analysis, including all groups except for the Uyghur (Figure 3). The Southern Han, Northern Han, Middle-Han, Min-nan, Taiwan aborigines, Miao, and Hui populations were clustered in one clade, while the remaining populations of the Hani and Bulang were clustered in another clade. The Middle Han population was closest to the Tujia population, followed by the Northern Han, the Min-nan, the Southern Han, the Miao, the Hui, and Taiwan aborigine populations. Compared with the Tujia populations, the Hani, Bulang, and the Uyghur populations were placed at the furthest distance. 


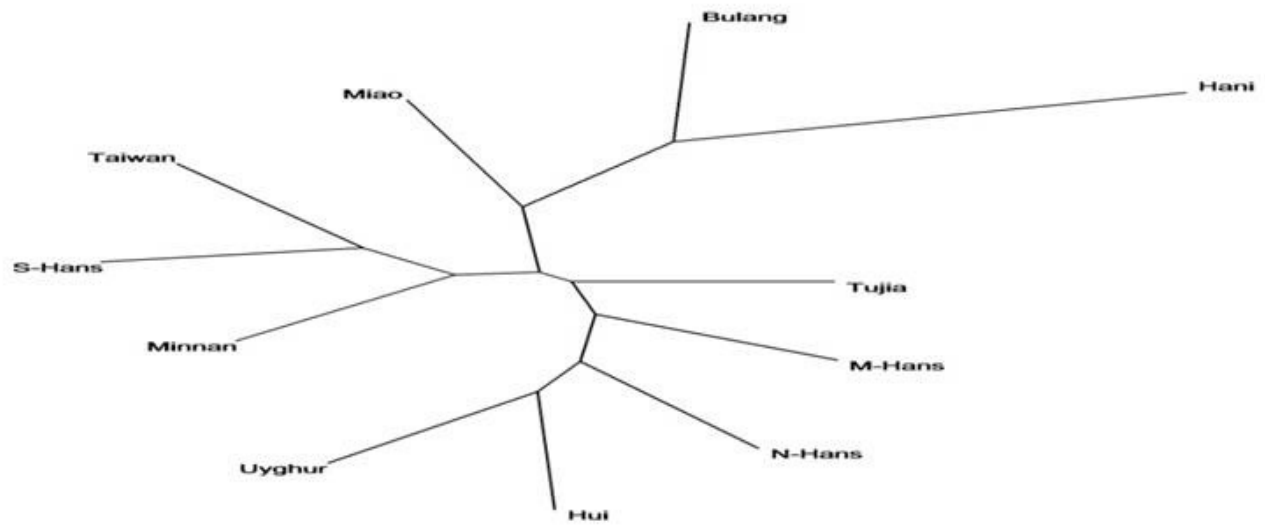

100

Figure 2. Dendrogram constructed by the neighbor-joining method showing the relationship between Tujia populations in Hubei with other populations in Chinese individuals of 10 regions based on the frequencies of HLADRB1 locus.

\section{Component Plot in Rotated Space}

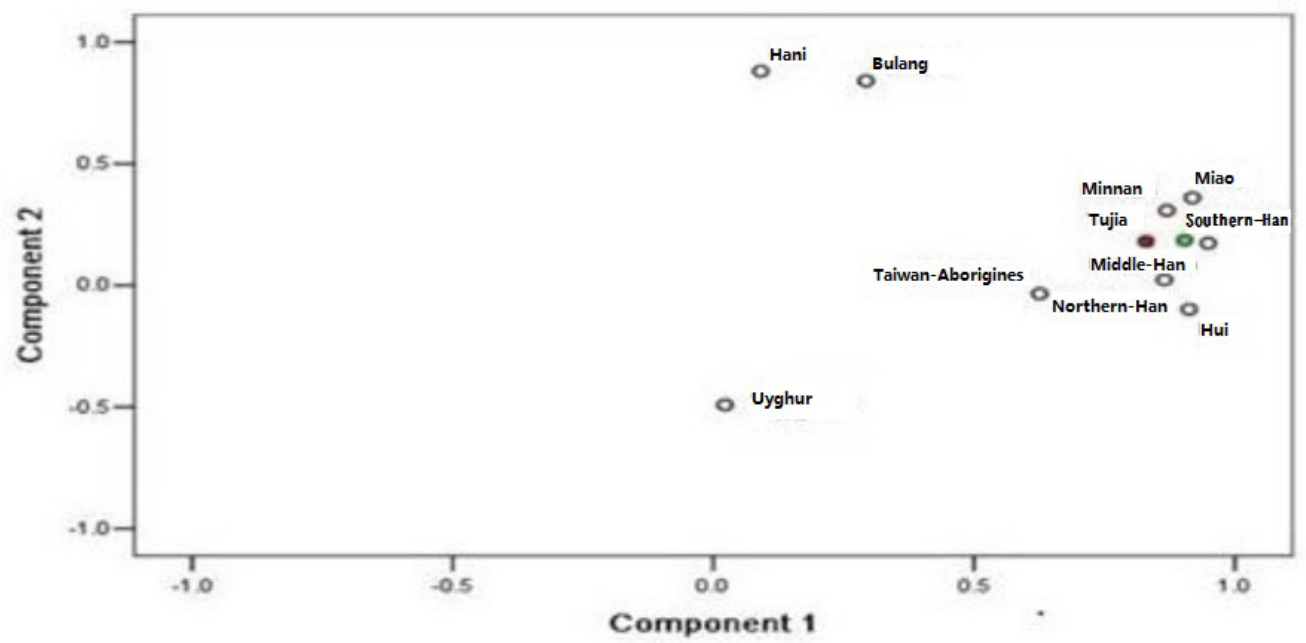

Figure 3. Principal component analysis between Tujia population in Hubei with Chinese of other 10 ethnic groups based on the allelic frequencies of HLA-DRB1 locus. 


\section{DISCUSSION}

The HLA gene family is classified into 3 groups, HLA-I, HLA-II, and HLA-III, according to gene polymorphisms, the distribution of the coding region, and function. Classic HLA-I group genes refer to 3 functional sites, including HLA-A, HLA-B, and HLA-C, which were the first members of the HLA gene family to be discovered (Petersdorf et al., 1999; Jin and Wang, 2003). Genes in the HLA-I group show a very high polymorphism rate; until recently, 1729 alleles of HLA-A, 2329 alleles of HLA-B, and 1291 alleles of HLA-C had been identified according to the International ImMunoGeneTics Information Project HLA database (http://www.ebi.ac.uk/imgt/hla/stats.html). HLA-I group gene products are mainly located on the surface of all nucleated cells, where they act in the presentation of foreign antigens to CD8 ${ }^{+}$ T-cells, enabling recognition and lysis of virus-infected cells (Maciag et al., 2000). The HLADR complex belongs to the classic HLA-II group; members of this group are mainly located on the surface of antigen-presenting cells and activated T-cells and their function is to present self-derived peptides to $\mathrm{CD}^{+}$T-cells (Rimmelzwaan et al., 2007). To date, 1051 alleles have been identified for the HLA-DR1 gene. Both HLA complex groups play very important roles in antigen recognition, immune response, and regulation (Stenger, 2001; Parham, 2005).

HLAs act as genetic markers of tumor susceptibility, and tumor-related studies are currently widely performed. Wufeng County in the Hubei Province shows a high incidence of cervical cancer, with an incidence rate of 1073.34 per 100,000 individuals, which is 423 times that of the lower incidence areas of Beijing $(2.54 / 100,000)$ and Shanghai $(3.80 / 100,000)$ (Ding and Ling, 2001). Hua et al. (2010) investigated the relationship between HLA-DRB1 allele polymorphisms and the susceptibility of Uyghur women to cervical carcinomas, and found that the frequency of HLA-DRB $1 * 15$ in women with cervical cancer was significantly higher than that in the control group $\left(\chi^{2}=4.05, \mathrm{P}<0.05\right.$, odds ratio $\left.=2.002\right)$, while the frequency of HLA-DRB1*04 was significantly lower than that in the control group $\left(\chi^{2}=7.283, \mathrm{P}<0.05\right.$, odds ratio $=0.432$ ). The HLA-DRB $1 * 15$ allele may be responsible for susceptibility to cervical cancer in Uyghur women from Kashgar, while the HLA-DRB1*04 allele may be a protective gene (Hua et al., 2010; Zhang et al., 2012b). Similarly, Ben Othmane et al. (2012) investigated HLA class II susceptibility to cervical cancer among Tunisian women. They found that the DRB $1 * 15$ allele alone was associated with a 2.7 -fold increase in risk for invasive cervical cancer. Matsumoto et al. (2012) demonstrated the protective effect of the DRB1*1302 allele against progression to cervical intraepithelial neoplasia 3 among Japanese women with lowgrade squamous intraepithelial lesions.

High rates of HLA polymorphisms indicate the diversity of genetic background among different areas of resident groups and different nationalities. The HLA genotype and allele frequency distribution between residents of different areas and different nationalities may show large variations. So before planning to use any genetic marker in disease-related research, basic studies should be performed to investigate the genetic background and establish a genetic database for different nationalities.

The distribution of HLA-DRB1 is related to ethnic origin and residency, as the HLA distribution differs significantly among different regions and ethnic groups. Some antigens also have ethnic specificity, such as A36, A43, B42, and DR18, which are almost exclusively present in black populations. A22, A2, B46, and B54, known as mongoloid antigens, are mainly present in Asian populations; however, B21, B41, and others are mainly present in 
Caucasians (Tan et al., 2006).

We previously reported the HLA-DRB1 allelic distributions in 124 unrelated healthy Tujia individuals (Zhang et al., 2012a). Among the HLA-DRB1 alleles, high frequencies of HLA-DRB1*09 (25.81\%) were observed, followed by HLA-DRB1*15 (12.9\%), DRB1*12 $(10.89 \%), \mathrm{DRB} 1 * 04(9.27 \%)$, and DRB1*08 (8.87\%). In this study, we increased the sample size and recruited 262 healthy, unrelated Tujia individuals. The aim was to further confirm the genetic diversity of HLA-DRB1 alleles in the Tujia population of Wufeng. Thirteen genotypes for HLA-DRB1 were detected; HLA-DRB1*09, DRB1*15, and DRB $1 * 12$ showed reduced in frequency compared with our previous data. However, the frequencies of HLA-DRB $1 * 08$ and DRB $1 * 04$ appeared to be increased (Zhang et al., 2012a). In this study, 13 genotypes of HLA-DRB1 were detected. Our results were comparable to corresponding data from the MHC database (dbMHC) of the Chinese population in the southeastern Asia region, which shows significant variation in the DRB1 genetic distribution. The 6 most common genotypes of HLA in Hubei Tujias are HLA-DRB1*09, DRB1*08, DRB1*15, DRB1*12, DRB1*04, and DRB1*14. Their corresponding gene frequencies are $22.52 \%, 11.07 \%(\mathrm{DRB} 1 * 08$ and DRB1*15), $10.69 \%(\mathrm{DRB} 1 * 12$ and $\mathrm{DRB} 1 * 04)$, and $7.82 \%$ (DRB1*14). According to data from dbMHC, all Chinese people inhabiting Southeastern Asia have the following 6 most common genotypes: DRB1*12, DRB1*14, DRB1*04, DRB1*08, DRB1*15, and DRB1*11, with corresponding gene frequencies of 17.4, 16.3, 12.9, 10.9, 10.9, and 10.2\% (http://www. ncbi.nlm.nih.gov/). A similarity between the Tujias and southeastern Chinese region population is the high gene frequency of DRB $1 * 15$, DRB $1 * 12$, DRB1*14, DRB1*08, and DRB1*04; one difference between the two populations is the frequency of DRB $1 * 09$, which showed a relatively low gene frequency among the Southeast Asian population, but presented a relatively high gene frequency in the Tujia population in this study. Conversely, DRB $1 * 11$, which has a relatively high gene frequency in the Southeastern Asian Chinese population, presented a relatively low gene frequency in the Tujias. This HLA-DRB1 distribution difference indicates that the HLA genotype differences are related to population ethnicity and regions of residence. The difference has manifested as a specific HLA genetic distribution during a long period of ethnic development and is important for understanding the genetic background, evolution, and ethnic origin of a population, as well as for applied research for studies related to genetics, the incidence of heritable diseases, and vaccine development.

The origin of the Tujia nationality is controversial; however, most researchers believe that Tujias are descendants of the ancient "Ba" nationality. It is thought that the most prosperous era for the Ba nationality, who inhabited Ba-Zi-Guo (now Chongqing area), was 400-600 $\mathrm{AD}$; however, Ba-Zi-Guo was invaded and conquered by the Qin (another ancient nation). The $\mathrm{Ba}$ people began to move eastwards from their original region (now the Chongqing region), and from 1300 BCE these eastward-migrated Ba people gradually came to be called "Tujia". Ancient Tujias, today inhabiting the west of Hunan and Hubei provinces, together with other nationalities in these areas, were referred to as "Wu-Yi-Man" or "Wu-Xi-Man" approximately 2000 years ago. After the Song dynasty (960-1279 BCE), the Tujia nationality was known as "Tu-Ding" or "Tu-Min"; they are now formally called the "Tujia Nationality" according to their wishes after the establishment of the Peoples' Republic of China. The oral Tujia language is an independent Bumar-Tibet language, which still belongs to the Chinese-Tibet language family. Tujias have no written language and use Chinese for writing. Currently, most Tujias can speak and write Chinese; however, the 200,000 Tujias who reside along the Qiu river 
(starting from Hefeng, Hubei Province, and ending at the Yuan river in Hunan Province) still predominantly speak the Tujia language, although some people can speak and write Chinese. Communication between the Tujias and the Chinese began in very ancient times and became more frequent after the late Qing dynasty.

By mapping the $\mathrm{Y}$ chromosome haplotype distribution in Tujia nationals, Xie et al. (2004) investigated the origin of the Tujia nationality and its communication with other nationalities. Their results indicated that the Tujia social structure is similar to the Chinese patriarchal structure. According to genetic relationships between the allelic frequencies of the HLA-DRB1 locus of Tujia nationals and those of another 10 ethnic groups (see Figure 2), the 11 groups can be divided into 2 clusters: 1) Miao, Taiwan-Minnan, Southern-Han, Hani, and Bulang, and 2) Northern Han, Hui, Tujia, Middle-Han, Taiwan-Aborigines, and Uyghur. Thus, the Tujia population is most closely related to the Middle-Han.

In addition, PCA showed that the Uyghur, Bulang, and Hani populations are distantly related to all other ethnic groups, perhaps because they live in remote areas and seldom communicate with other groups. Our study further indicates that the Hubei Tujia nationality (the north branch of the Tujia nationality) has high similarity with the Central Chinese population with regard to HLA-DRB1 allele frequency, which also indicates that the two populations have similar genetic structures and blood relationships.

\section{ACKNOWLEDGMENTS}

Research supported by the National Nature Science Foundation of China (\#30872935 and \#81072123).

\section{REFERENCES}

Ben Othmane Y, Ghazouani E, Mezlini A, Lagha A, et al. (2012). HLA class II susceptibility to cervical cancer among Tunisian women. Bull. Cancer 99: 81-86.

Bodmer WF (1987). The HLA system: structure and function. J. Clin. Pathol. 40: 948-958.

Chen S, Ren X, Liu Y, Hu Q, et al. (2007). Human leukocyte antigen class I polymorphism in Miao, Bouyei, and Shui ethnic minorities of Guizhou, China. Hum. Immunol. 68: 928-933.

Ding M and Ling X (2001). Advance in epidemiology and etiology of cervical cancer. J. Pract. Obstet. Gynecol. 17: 61-62.

Excoffier L and Slatkin M (1995). Maximum-likelihood estimation of molecular haplotype frequencies in a diploid population. Mol. Biol. Evol. 12: 921-927.

Hua L, Bahtinsha A, Suqin Z and Guzalnur A (2010). The relationships between HLA-DRB1*15 or HLA-DRB1*04 and cervical cancer in Kashgar Uyghur women. Fudan Univ. J. Med. Sci. 37: 555-559.

Ivanova M, Rozemuller E, Tyufekchiev N, Michailova A, et al. (2002). HLA polymorphism in Bulgarians defined by highresolution typing methods in comparison with other populations. Tissue Antigens 60: 496-504.

Jin P and Wang E (2003). Polymorphism in clinical immunology - From HLA typing to immunogenetic profiling. $J$. Transl. Med. 1: 8 .

Maciag PC, Schlecht NF, Souza PS, Franco EL, et al. (2000). Major histocompatibility complex class II polymorphisms and risk of cervical cancer and human papillomavirus infection in Brazilian women. Cancer Epidemiol. Biomarkers Prev. 9: 1183-1191.

Matsumoto K, Maeda H, Oki A, Takatsuka N, et al. (2012). HLA class II DRB1*1302 allele protects against progression to cervical intraepithelial neoplasia grade 3: a multicenter prospective cohort study. Int. J. Gynecol. Cancer 22: 471-478.

Parham P (2005). MHC class I molecules and KIRs in human history, health and survival. Nat. Rev. Immunol. 5: 201-214.

Petersdorf EW, Mickelson EM, Anasetti C, Martin PJ, et al. (1999). Effect of HLA mismatches on the outcome of hematopoietic transplants. Curr. Opin. Immunol. 11: 521-526.

Qiu X, Zhang F, Chen D, Azad AK, et al. (2011). HLA-B*07 is a high risk allele for familial cervical cancer. Asian Pac. J. Cancer Prev. 12: 2597-2600. 
Rimmelzwaan GF, Fouchier RA and Osterhaus AD (2007). Influenza virus-specific cytotoxic T lymphocytes: a correlate of protection and a basis for vaccine development. Curr. Opin. Biotechnol. 18: 529-536.

Saitou N and Nei M (1987). The neighbor-joining method: a new method for reconstructing phylogenetic trees. Mol. Biol. Evol. 4: 406-425.

Shankarkumar U, Sridharan B and Pitchappan RM (2003). HLA diversity among Nadars, a primitive Dravidian caste of South India. Tissue Antigens 62: 542-547.

Shaw CK, Chen LL, Lee A and Lee TD (1999). Distribution of HLA gene and haplotype frequencies in Taiwan: a comparative study among Min-nan, Hakka, Aborigines and Mainland Chinese. Tissue Antigens 53: 51-64.

Shen CM, Zhu BF, Deng YJ, Ye SH, et al. (2010). Allele polymorphism and haplotype diversity of HLA-A, -B and -DRB1 loci in sequence-based typing for Chinese Uyghur ethnic group. PLoS One 5: e13458.

Shi L, Shi L, Yao YF, Matsushita M, et al. (2010). Genetic link among Hani, Bulang and other Southeast Asian populations: evidence from HLA -A, -B, -C, -DRB1 genes and haplotypes distribution. Int. J. Immunogenet. 37: 467-475.

Stenger S (2001). Cytolytic T cells in the immune response to mycobacterium tuberculosis. Scand. J. Infect Dis. 33: 483487.

Tan JM, Zhou YC and Tang CD (2006). Technology and Clinical Applications of Tissue Matching. People's Health Publishing House, Beijing, 86-88.

Xie XH, Li H, Mao XY, Wen B, et al. (2004). Genetic structure of Tujia as revealed by Y chromosomes. Yi Chuan Xue Bao 31: 1023-1029.

Zhang L, Cheng D, Tao N, Zhao M, et al. (2012a). Distribution of HLA-A, -B and -DRB1 genes and haplotypes in the Tujia population living in the Wufeng Region of Hubei Province, China. PLoS One 7: e38774.

Zhang SQ, Lalai S, Guzalnur A, Hamragul A, et al. (2012b). Distribution of HLA-DRB1 allele polymorphism in the Uyghur women with family history of cervical cancer. Zhonghua Zhong Liu Za Zhi 34: 272-277. 